\title{
An extension of a criterion for unimodality
}

\author{
Jenny Alvarez \\ Department of Mathematics \\ UC Santa Barbara, CA, USA \\ jalvar01@umail.ucsb.edu
}

\author{
Miguel Amadis \\ Department of Mathematics \\ Nyack College, New York, NY, USA \\ amadism@nyack.edu
}

\author{
George Boros \\ Department of Mathematics \\ Xavier University, New Orleans, LA 70125 USA \\ gboros@xula.edu \\ Dagan Karp \\ Department of Mathematics \\ Tulane University, New Orleans, LA 70118 USA \\ dkarp@math . tulane .edu \\ Victor H. Moll \\ Leobardo Rosales \\ Department of Mathematics \\ Tulane University, New Orleans, LA 70118 USA \\ vhm@math.tulane.edu \\ Department of Mathematics \\ UC San Diego, CA USA \\ Irosales@ucsd.edu
}

Submitted: March 20, 2001; Accepted: September 19, 2001.

Subject Classifications: 40, 33, 05

\begin{abstract}
We prove that if $P(x)$ is a polynomial with nonnegative nondecreasing coefficients and $n$ is a positive integer, then $P(x+n)$ is unimodal. Applications and open problems are presented.
\end{abstract}

\section{Introduction}

A finite sequence of real numbers $\left\{d_{0}, d_{1}, \cdots, d_{m}\right\}$ is said to be unimodal if there exists an index $0 \leq m^{*} \leq m$, called the mode of the sequence, such that $d_{j}$ increases up to $j=m^{*}$ and decreases from then on, that is, $d_{0} \leq d_{1} \leq \cdots \leq d_{m^{*}}$ and $d_{m^{*}} \geq d_{m^{*}+1} \geq \cdots \geq d_{m}$. A polynomial is said to be unimodal if its sequence of coefficients is unimodal. 
Unimodal polynomials arise often in combinatorics, geometry and algebra. The reader is referred to [3] and [4] for surveys of the diverse techniques employed to prove that specific families of polynomials are unimodal.

A sequence of positive real numbers $\left\{d_{0}, d_{1}, \cdots, d_{m}\right\}$ is said to be logarithmic concave (or log concave for short) if $d_{j+1} d_{j-1} \leq d_{j}^{2}$ for $1 \leq j \leq m-1$. It is easy to see that if a sequence is $\log$ concave then it is unimodal [5]. A sufficient condition for log concavity of a polynomial is given by the location of its zeros: if all the zeros of a polynomial are real and negative, then it is log concave and therefore unimodal [5]. A simple criterion for unimodality was established in [2]: if $a_{j}$ is a nondecreasing sequence of positive real numbers, then

$$
\begin{aligned}
P(x+1) & =\sum_{j=0}^{m} a_{j}(x+1)^{j} \\
& =\sum_{j=0}^{m} d_{j}(m) x^{j}
\end{aligned}
$$

is unimodal. This criterion is reminiscent of Brenti's criterion for log concavity [3]. A sequence of real numbers is said to have no internal zeros if $d_{i}, d_{k} \neq 0$ and $i<j<k$ imply $d_{j} \neq 0$. Brenti's criterion states that if $P(x)$ is a $\log$ concave polynomial with nonnegative coefficients and with no internal zeros, then $P(x+1)$ is log concave.

In this paper we first prove that under the same conditions of [2] the polynomial $P(x+n)$ is unimodal for any $n \in \mathbb{N}$, the set of positive integers. We also characterize the unimodal sequences $\left\{d_{j}\right\}$ that appear in [2] and discuss the behavior of the coefficients of $P(x+1)$ for a unimodal polynomial $P(x)$. Numerical evidence suggests that the unimodality result is true for $n$ real and positive. This remains to be investigated.

\section{The extension}

In this section we prove an extension of the main result in [2]. We start by establishing an elementary inequality.

Lemma 2.1 Let $m, n \in \mathbb{N}$ and $m_{*}:=\left\lfloor\frac{m}{n+1}\right\rfloor$. Then $(n+1) m_{*} \leq m \leq(n+1) m_{*}+n$.

Proof This follows directly from $\frac{m}{n+1}-1<m_{*} \leq \frac{m}{n+1}$.

Theorem 2.2 Let $0 \leq a_{0} \leq a_{1} \cdots \leq a_{m}$ be a sequence of real numbers and $n \in \mathbb{N}$, and consider the polynomial

$$
P(x)=a_{0}+a_{1} x+a_{2} x^{2}+\cdots+a_{m} x^{m} .
$$

Then the polynomial $P(x+n)$ is unimodal with mode $m_{*}=\left\lfloor\frac{m}{n+1}\right\rfloor$. 
We now restate Theorem 2.2 in terms of the coefficients of $P$.

Theorem 2.3 Let $0 \leq a_{0} \leq a_{1} \cdots \leq a_{m}$ be a sequence of real numbers and $n \in \mathbb{N}$. Then the sequence

$$
q_{j}:=q_{j}(m, n)=\sum_{k=j}^{m} a_{k}\left(\begin{array}{l}
k \\
j
\end{array}\right) n^{k-j}
$$

is unimodal with mode $m_{*}=\left\lfloor\frac{m}{n+1}\right\rfloor$.

Proof The coefficients $q_{j}(m)$ in (2) are given by

$$
q_{j}(m)=\sum_{k=j}^{m} a_{k}\left(\begin{array}{l}
k \\
j
\end{array}\right) n^{k-j}
$$

so that Theorem 2.3 follows from Theorem 2.2. Now

$$
(i+1)\left(q_{i+1}(m)-q_{i}(m)\right) \leq \sum_{k=i}^{m} a_{k}\left(\begin{array}{c}
k \\
i
\end{array}\right) n^{k-i-1}[k-(n+1) i-n] .
$$

Suppose $m_{*} \leq i \leq m-1$. Then

$$
k-(n+1) i-n \leq m-(n+1) i-n \leq m-(n+1) m_{*}-n \leq 0,
$$

where we have employed the Lemma in the last step. We conclude that every term in the sum (4) is nonpositive. Thus for $m_{*} \leq i \leq m-1$ we have $q_{i+1}(m) \leq q_{i}(m)$.

Now assume $0 \leq i \leq m_{*}-1$. We show that $q_{i+1}(m) \geq q_{i}(m)$. Observe that in this case the sum (4) contains terms of both signs, so the positivity of the sum is not apriori clear. Consider

$$
\begin{aligned}
(i+1)\left(q_{i+1}(m)-q_{i}(m)\right)= & \sum_{k=(n+1) i+n+1}^{m} a_{k}\left(\begin{array}{c}
k \\
i
\end{array}\right) n^{k-i-1}[k-(n+1) i-n] \\
& -\sum_{k=i}^{(n+1) i+n-1} a_{k}\left(\begin{array}{c}
k \\
i
\end{array}\right) n^{k-i-1}[-k+(n+1) i+n] \\
:= & T_{2}-T_{1} .
\end{aligned}
$$

Observe that

$$
\begin{aligned}
T_{1} & =\sum_{k=i}^{(n+1) i+n-1} a_{k}\left(\begin{array}{c}
k \\
i
\end{array}\right) n^{k-i-1}[-k+(n+1) i+n] \\
& \leq a_{(n+1)(i+1)} \sum_{k=i}^{(n+1) i+n-1}\left(\begin{array}{c}
k \\
i
\end{array}\right) n^{(n+1) i+n-1-i-1}[-k+(n+1) i+n] \\
& \leq a_{(n+1)(i+1)} n^{(i+1) n-2} \sum_{k=i}^{(n+1) i+n-1}\left(\begin{array}{c}
k \\
i
\end{array}\right)[-k+(n+1) i+n] .
\end{aligned}
$$


The monotonicity of the coefficients of $P$ was used in the first step.

The last sum can be evaluated (e.g. symbolically) as

$$
\sum_{k=i}^{(n+1) i+n-1}\left(\begin{array}{l}
k \\
i
\end{array}\right)[-k+(n+1) i+n]=\frac{((n+1) i+n+1) !}{(i+2) !(n i+n-1) !},
$$

so that

$$
\begin{aligned}
T_{1} & \leq a_{(n+1)(i+1)} n^{(i+1) n} \times \frac{((n+1) i+n+1) !}{n^{2}(i+2) !(n i+n-1) !} \\
& \leq a_{(n+1)(i+1)} n^{(i+1) n} \times \frac{((n+1) i+n+1) !}{(n i+2 n)(n i+n) i !(n i+n-1) !} .
\end{aligned}
$$

Now observe that

$$
\frac{((n+1) i+n+1) !}{(n i+2 n)(n i+n) i !(n i+n-1) !} \leq\left(\begin{array}{c}
(n+1)(i+1) \\
i
\end{array}\right) .
$$

The inequality $T_{1} \leq T_{2}$ now follows since the upper bound for $T_{1}$ established above is the first term in the sum defining $T_{2}$.

Corollary 2.4 Let $0 \leq a_{0} \leq a_{1} \cdots \leq a_{m}$ be a sequence of real numbers, $n \in \mathbb{N}$, and

$$
P(x)=a_{0}+a_{1} x+a_{2} x^{2}+\cdots+a_{m} x^{m} .
$$

Then $P(x+n)$ has decreasing coefficients for $n \geq m$.

Example 2.5 Let $2<a_{1}<\cdots<a_{p}$ and $r_{1}, \cdots, r_{p}$ be two sequences of positive integers. Then the sequence

$$
q_{j}:=\sum_{k=j}^{m} n^{k-j}\left(\begin{array}{c}
a_{1} m \\
k^{r_{1}}
\end{array}\right)\left(\begin{array}{c}
a_{2} m \\
k^{r_{2}}
\end{array}\right) \cdots\left(\begin{array}{c}
a_{p} m \\
k^{r_{p}}
\end{array}\right)\left(\begin{array}{l}
k \\
j
\end{array}\right), \quad 0 \leq j \leq m
$$

is unimodal.

\section{The converse of the original criterion}

The original criterion for unimodality states that if $P(x)$ has positive nondecreasing coefficients, then $P(x+1)$ is unimodal. In this section we discuss the following inverse question:

Given a unimodal sequence $\left\{d_{j}: 0 \leq j \leq m\right\}$, is there a polynomial $P(x)=a_{0}+a_{1} x+$ $\cdots+a_{m} x^{m}$ with nonnegative nondecreasing coefficients such that

$$
P(x+1)=\sum_{j=0}^{m} d_{j} x^{j}
$$


We begin by expressing the conditions on $\left\{a_{j}\right\}$ that guaranteed unimodality of $P(x+1)$ in terms of the coefficients $\left\{d_{j}\right\}$. Recall that

$$
d_{j}=\sum_{k=j}^{m} a_{k}\left(\begin{array}{l}
k \\
j
\end{array}\right)
$$

and

$$
a_{j}=\sum_{k=j}^{m}(-1)^{k-j} d_{k}\left(\begin{array}{l}
k \\
j
\end{array}\right)
$$

Lemma 3.1 Let $0 \leq j \leq m$. Then

$$
a_{j} \geq 0 \Longleftrightarrow d_{j} \geq \sum_{k=j+1}^{m}(-1)^{k-j+1} d_{k}\left(\begin{array}{l}
k \\
j
\end{array}\right)
$$

Proof This follows directly from (3).

Lemma 3.2 Let $0 \leq j \leq m-1$. Then

$$
a_{j} \leq a_{j+1} \Longleftrightarrow d_{j} \leq \sum_{k=j+1}^{m}(-1)^{k-j+1} d_{k}\left(\begin{array}{c}
k+1 \\
j+1
\end{array}\right) .
$$

Proof This follows directly from the identity

$$
a_{j+1}-a_{j}=\sum_{k=j+1}^{m}(-1)^{k-j+1} d_{k}\left(\begin{array}{c}
k+1 \\
j+1
\end{array}\right)-d_{j} .
$$

We now combine the previous two lemmas to produce a criterion for unimodality.

Theorem 3.3 Let $Q(x)=d_{0}+d_{1} x+\cdots+d_{m} x^{m}$ and assume the coefficients $\left\{d_{j}\right\}$ satisfy the inequalities

$$
\sum_{k=j+1}^{m}(-1)^{k-j+1} d_{k}\left(\begin{array}{l}
k \\
j
\end{array}\right) \leq d_{j} \leq \sum_{k=j+1}^{m}(-1)^{k-j+1} d_{k}\left(\begin{array}{l}
k+1 \\
j+1
\end{array}\right) .
$$

Then $Q(x)$ is a unimodal polynomial for which $P(x):=Q(x-1)$ has positive and nondecreasing coefficients. Furthermore, for any $n \in \mathbb{N}, Q(x+n)$ is unimodal with mode $\left\lfloor\frac{m}{n+2}\right\rfloor$.

Proof The first part follows from the previous two lemmas. For the second part, Theorem 3.3 shows that $Q(x-1)$ has nonnegative, nondecreasing coefficients, so Theorem 2.2 yields the result. 
Note. The inequality (5) is always consistent. The difference between the upper and lower bound is

$$
\begin{gathered}
\sum_{k=j+1}^{m}(-1)^{k-j+1} d_{k}\left(\begin{array}{c}
k+1 \\
j+1
\end{array}\right)-\sum_{k=j+1}^{m}(-1)^{k-j+1} d_{k}\left(\begin{array}{l}
k \\
j
\end{array}\right) \\
=\sum_{k=j+1}^{m}(-1)^{k-j+1} d_{k}\left(\begin{array}{c}
k \\
j+1
\end{array}\right)=a_{j+1},
\end{gathered}
$$

so the difference is always nonnegative.

Note. It would be interesting to describe the precise range of the map $\left(a_{0}, a_{1}, \cdots, a_{m}\right) \mapsto$ $\left(d_{0}, d_{1}, \cdots, d_{m}\right)$. This map is linear, so the image of the set $0 \leq a_{0} \leq \cdots \leq a_{m}$ is a polyhedral cone. In this paper we state one simple restriction on this image.

Proposition 3.4 Let $a_{j} \geq 0$. Then $d_{j} \geq d_{j+1}$ for $j \geq\lfloor m / 2\rfloor$.

Proof This follows directly from

$$
\begin{aligned}
d_{j}-d_{j+1} & =\sum_{k=j}^{m} a_{k}\left(\begin{array}{c}
k \\
j
\end{array}\right)-\sum_{k=j+1}^{m} a_{k}\left(\begin{array}{c}
k \\
j+1
\end{array}\right) \\
& =a_{j}+\sum_{k=j+1}^{m} a_{k} \frac{k !(2 j+1-k)}{(j+1) !(k-j) !}
\end{aligned}
$$

since every term in the last sum is nonnegative.

\section{A criterion for log concavity}

Any nonnegative differentiable function $f$ that satisfies $f(0)=f(m)=0$ and $f^{\prime \prime}(x) \leq 0$ yields the unimodal sequence $\{f(j): 0 \leq j \leq m\}$. The next theorem shows that these sequences are always log concave.

Proposition 4.1 Let $P(x)=\sum_{k=0}^{m} c_{k} x^{k}$ be a unimodal polynomial with mode $n$. Assume in addition that $c_{j+1}-2 c_{j}+c_{j-1} \leq 0$. Then $P(x)$ is log concave.

Proof Let $j<n$, so that $c_{j} \geq c_{j-1}$. The condition on $c_{j}$ can be written as $c_{j}-c_{j-1} \geq$ $c_{j+1}-c_{j}$, so that

$$
c_{j} c_{j}-c_{j} c_{j-1} \geq c_{j+1} c_{j-1}-c_{j} c_{j-1},
$$

and thus the $\log$ concavity condition holds. The case $j \geq n$ is similar. 


\section{The motivating example}

The original criterion for unimodality in [2] was developed in our study of the coefficients $d_{l}(m)$ of the polynomial

$$
P_{m}(a)=\frac{1}{\pi} 2^{m+3 / 2}(a+1)^{m+1 / 2} \int_{0}^{\infty} \frac{d x}{\left(x^{4}+2 a x^{2}+1\right)^{m+1}}
$$

considered in [1]. These coefficients are given explicitly by

$$
d_{l}(m)=2^{-2 m} \sum_{k=l}^{m} 2^{k}\left(\begin{array}{c}
2 m-2 k \\
m-k
\end{array}\right)\left(\begin{array}{c}
m+k \\
m
\end{array}\right)\left(\begin{array}{l}
k \\
l
\end{array}\right),
$$

and we have conjectured that $\left\{d_{l}(m)\right\}_{l=0}^{m}$ forms a log concave sequence. Unfortunately Proposition 4.1 does not settle this question. For example, for $m=15$ the sequence of signs in $d_{j+1}(15)-2 d_{j}(15)+d_{j-1}(15)$, for $1 \leq j \leq 14$, is

$$
\operatorname{sign}(15)=\{+1,+1,+1,+1,+1,-1,-1,-1,-1,+1,+1,+1,+1,+1\},
$$

so the condition fails.

\section{Acknowledgements}

The work presented here was part of a SIMU project at the University of Puerto Rico at Humacao. The authors wish to thank Herbert Medina and Ivelisse Rubio for bringing them together. The fifth author acknowledges the partial support of NSF-DMS 0070567, Project number 540623.

\section{References}

[1] Boros, G. - Moll, V.: An integral hidden in Gradshteyn and Rhyzik. Jour. Comp. Appl. Math. 237, 272-287, 1999.

[2] Boros, G. - Moll, V.: A criterion for unimodality. Elec. Jour. Comb. 6, \# R10, 1999.

[3] Brenti, F.: Log-concave and unimodal sequences in Algebra, Combinatorics and Geometry: an update. Contemporary Mathematics, 178, 71-84, 1994.

[4] Stanley, R.: Log-concave and unimodal sequences in algebra, combinatorics and geometry. Graph theory and its applications: East and West (Jinan, 1986), 500-535, Ann. New York Acad. Sci., 576, New York, 1989.

[5] Wilf, H.S.: generatingfunctionology. Academic Press, 1990. 